\title{
Precision measurements with kaonic atoms - from DEAR to SIDDHARTA
}

\author{
J. Zmeskal on behalf of the DEAR and SIDDHARTA \\ collaboration
}

Stefan Meyer Institut, ÖAW, Vienna, Austria

\begin{abstract}
The final results of the DEAR (DAФNE Exotic Atom Research) experimental program at Laboratori Nazionali di Frascati [1] to measure the strong interaction induced shift and width of the kaonic hydrogen 1s atomic state will be presented, together with an overview of the target and detector system. The hadronic shift and width of the 1s state are sensitive observables to study quantitatively the low energy antikaon-nucleon interaction.

Proceeding to a precise determination of the isospin-dependent antikaon-nucleon scattering lengths a new detector has to be developed, namely, a Silicon Drift Detector (SDD) with good timing properties and excellent energy resolution. This new SDD system together with a kaon-trigger will suppress the $\mathrm{X}$-ray background by more then two orders of magnitude compared to the DEAR case and thus will allow to measure kaonic deuterium X-rays for the first time.

Kaonic hydrogen together with precision measurements of kaonic helium Xrays will allow the study of the sub-threshold $\Lambda(1405)$ resonance, which might lead to strongly bound kaonic states in light nuclei. Furthermore, these precision measurements are a powerful tool to test chiral symmetry breaking in systems with strangeness.
\end{abstract}

\section{Introduction}

This research deals with the characteristic properties of the strong interaction of antikaons $\left(\mathrm{K}^{-}\right)$with nucleons $(\mathrm{p})$. These hadrons consist of light quarks and have masses which are considerably larger than the sum of their current quark masses. This extraordinary phenomenon is proposed to originate from spontaneous breaking of chiral symmetry of massless quarks in strong interaction physics [2]. It results in a ground state - the vacuum state - with a finite expectation value of quark-antiquark pairs, the chiral quark condensate [3]. The hadrons are considered to be quasiparticle excitations of this chiral 
condensate. The lowest excitation modes of the condensate, so called Nambu-Goldstone bosons [2], are identified as mesons containing light quarks. Their s-wave interaction with nucleons is determined by the pion and kaon decay constants $\mathrm{f}_{\pi}$ and $\mathrm{f}_{\mathrm{K}}$ respectively [4], the squares of which are also the order parameters of chiral symmetry breaking $[5,6]$ and are directly connected to the magnitude of the quark condensate through the Gell-MannOaks-Renner relation [6].

With precise X-ray spectroscopy on kaonic hydrogen, the chiral symmetry breaking scenario in the strangeness sector will be investigated by studying the $\mathrm{K}^{-} \mathrm{p}$ and $\mathrm{K}^{-} \mathrm{d}$ s-wave interaction at threshold. In a previous experiment $[7,8]$ a repulsive strong interaction shift of the 1s state in kaonic hydrogen was found, in agreement with scattering data and in disagreement with earlier results obtained in X-ray measurements. With better precision this finding was also confirmed by the result of DEAR $[9,10]$. The origin of this repulsive strong interaction at threshold can be traced back to the presence of the $\Lambda(1405)$ resonance which leads on one hand to a repulsive $K^{-} p$ scattering length [11] and on the other to the possible existence of strongly bound kaonic states in light nuclei [12]. Very recently, searches for such exotic kaonic cluster states were performed and evidence has been reported for a ppnK ${ }^{-}$state with a binding energy of about $170 \mathrm{MeV}$ and a width of about $20 \mathrm{MeV}$ [13]. An indication of the formation of kaonic ${ }^{3} \mathrm{He}$ with similar binding energy has been found in $1.9 \mathrm{~A} \mathrm{GeV} \mathrm{Ni-Ni} \mathrm{collision} \mathrm{[14].} \mathrm{These} \mathrm{findings} \mathrm{are} \mathrm{in} \mathrm{line} \mathrm{with}$ results of enhanced sub-threshold production of $\mathrm{K}^{-}$mesons in heavy ion collisions, indicating a reduced $\mathrm{K}^{-}$mass in the dense medium of the collision zone [15].

In view of these developments and the fundamental importance of the s-wave $\mathrm{K}^{-} \mathrm{N}$ scattering length for chiral dynamics with strangeness, it is of utmost importance to perform precise experiments and determine the strong interaction shift and width of kaonic hydrogen and deuterium and also of ${ }^{4} \mathrm{He}$ as well as ${ }^{3} \mathrm{He}[16]$.

\section{The DEAR experimental setup}

The DEAR experiment uses low momentum negative kaons, produced by the decay of the $\Phi$ meson at DA $\Phi$ NE, the $\Phi$-factory at Laboratori Nazionale di Frascati. The kaons leave the interaction point through the DEAR beam pipe made out of thin-walled aluminum with carbon fiber reinforcement. They are degraded in energy to a few $\mathrm{MeV}$ and enter a gaseous hydrogen target placed above the beam pipe where they are stopped (Fig 1). A light-weight cryogenic target was built, consisting of aluminum (top-plate and entrance-ring), Kapton (side wall and entrance window) and a support structure of fiberglas reinforced epoxy to minimize fluorescence X-rays produced in the target material. The target cell is cooled to $25 \mathrm{~K}$ with a two stage closed-cycle helium refrigerator. The vacuum requirement is fulfilled using a wide range turbomolecular pump (TMP) with an oil-free diaphragm pump as forepump.

16 CCD detector chips (Marconi Applied Technologies, CCD55-30) with a total area of $116 \mathrm{~cm}^{2}$ were placed around the cryogenic target cell. Each chip has $1242 \times 1152$ pixels with a pixel size of $22.5 \mu \mathrm{m} \times 22.5 \mu \mathrm{m}$ and a depletion depth of about $30 \mu \mathrm{m}$. An energy resolution of $150 \mathrm{eV}$ at $6 \mathrm{keV}$ was achieved at a working temperature of $165 \mathrm{~K}$ using a read out time of 90 seconds. Sophisticated shielding of the DEAR target and 
detector was developed through different test runs at DA $\Phi$ NE. Finally, a graded shielding structure was used, starting with lead, followed by a copper and an aluminum layer, with the innemost layer of polycarbonate. With this setup and an improved beam setting, achieved by the DA $\Phi$ NE machine staff, the bremsstrahlung background could be reduced drastically, thereby enabling the kaonic hydrogen measurement.

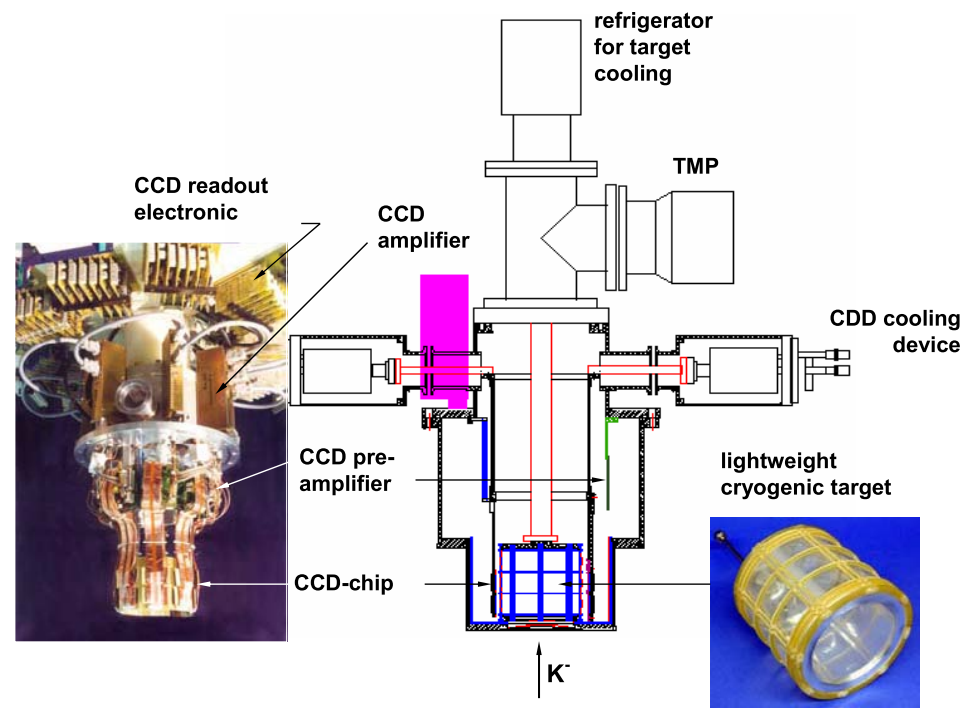

Figure 1: A sketch of the final DEAR apparatus.

\section{Kaonic nitrogen, DEAR final results}

Three sequential X-ray lines from kaonic nitrogen transitions [17] were measured and the corresponding X-ray yields obtained. This measurement was done on one hand to optimize the target detector system for the kaonic hydrogen run, on the other hand it is interesting for its own. Cascade processes (Auger transitions, Coulomb de-excitation, elastic scattering) and electron refilling (screening) are not yet well understood, leading to problems, for example, in the determination of the charged kaon mass. The main uncertainty in the determination of the charged kaon mass by measuring kaonic X-ray energies results from the not yet well known electron refilling process. The most precise determination of the charged kaon mass (determined from kaonic X-ray energy measurements) by two experimental groups shows a difference in their reported values of $60 \mathrm{keV}$, which dominates the error in the $\mathrm{K}^{-}$mass given by the PDG [18]. With our measurement we were able to show that kaonic nitrogen atoms are a highly ionized system [19], and therefore a precise determination of the charged kaon mass, as we proposed [20], will be possible without theoretical uncertainty in the energy shift due electron screening (refilling). 


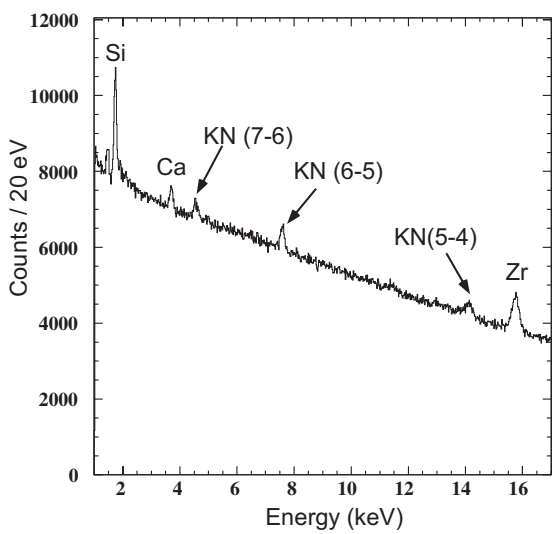

Figure 2: The three kaonic nitrogen lines are clearly visible. The fluorescence X-rays from Si $(1.74 \mathrm{keV}), \mathrm{Ca}(3.69 \mathrm{keV})$ and $\mathrm{Zr}(15.78+15.69 \mathrm{keV})$ are used for in-situ energy calibration and controlling of the energy resolution.

Table 1: Summary of the results of the kaonic nitrogen analysis [17]

\begin{tabular}{c|c|c|c}
\hline \hline Transition & $7 \rightarrow 6$ & $6 \rightarrow 5$ & $5 \rightarrow 4$ \\
\hline Calculated energy (keV) & 4.5773 & 7.5957 & 13.996 \\
\hline $\begin{array}{c}\text { Measured number of } \\
\text { events }\left(\times 10^{3}\right)\end{array}$ & $3.31 \pm 0.69$ & $5.28 \pm 0.38$ & $1.21 \pm 0.32$ \\
\hline $\begin{array}{c}\text { Predicted number of } \\
\text { events }\left(\times 10^{3}\right)\end{array}$ & $7.97 \pm 0.79$ & $9.59 \pm 0.96$ & $2.10 \pm 0.21$ \\
\hline Yield $(\%)$ & $41.5 \pm 8.7 \pm 4.1$ & $55.0 \pm 3.9 \pm 5.5$ & $57.4 \pm 15.2 \pm 5.7$ \\
\hline \hline
\end{tabular}

\section{Kaonic hydrogen, DEAR final results}

The experimental challenge of DEAR was the extraction of a small signal in the presence of a large low-energy X-ray background mainly from electron gamma showers resulting from lost electrons and positrons due to either Touschek scattering or beam interactions with residual gas, Fig. 3a. The careful optimization of the shielding of our experimental setup and the improvements in the beam optics achieved by the machine crew made this first and precise measurement of kaonic hydrogen at DA $\Phi$ NE possible.

The linearity of the CCD detector as well as the energy stability was measured insitu using titanium and zirconium fluorescence X-ray lines, coming from foils placed on top of the target cell. By fixing the energy position of the $\mathrm{Ti}$ and $\mathrm{Zr}$ lines, the position of the $\mathrm{Ca}$ line could be determined with accuracy of better than $1 \mathrm{eV}$. In addition, the resolution of the $\mathrm{Ti} \mathrm{K}_{\alpha}$ line width for the sum of all CCDs was better than $150 \mathrm{eV}$ for the whole beam 
time.

Although all material used for the target cell and the mounting device for the CCDs was carefully checked using different analysis methodes, it was not possible to completely avoid iron impurities. The iron fluorescence line overlaps partly with the kaonic hydrogen $\mathrm{K}_{\alpha}$ line and has to be disentangled. Based on two background measurements - the first with kaonic nitrogen as target gas, the second with hydrogen, but separated beams (meaning no collisions and therefore no kaons produced) - it was possible to subtract the iron fluorescence line from the kaonic $\mathrm{K}_{\alpha}$ line. After subtraction of the continuous background

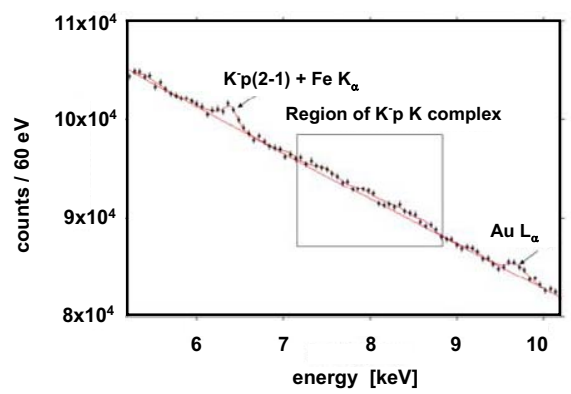

a)

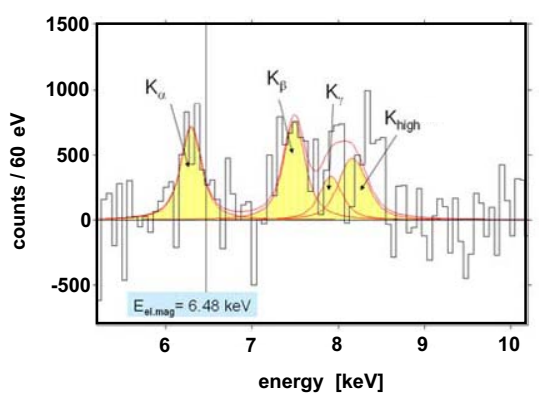

b)

Figure 3: a) Kaonic hydrogen X-ray spectrum for an integrated luminosity of $60 \mathrm{pb}^{-1} \mathrm{using}$ only single and double pixel events, showing the large bremsstrahlung background coming from Touschek effect and beam gas interaction. b) Continuous background and fluorescence X-ray line subtracted kaonic hydrogen spectrum.

and the iron fluorescence line (Fig. 3b) the data were fitted with the position and width of the $\mathrm{K}_{\alpha}$ line as free parameter and the constraint that $\mathrm{K}_{\beta}$ and $\mathrm{K}_{\gamma}$ have the same line width and fixed position relative to the $\mathrm{K}_{\alpha}$ line, as given by QED calculations.

The result of DEAR for the 1s strong interaction shift and absorption width of kaonic hydrogen is $[9,10]$ :

$$
\begin{aligned}
\epsilon_{1 \mathrm{~s}} & =-193 \pm 37 \text { (stat.) } \pm 6 \text { (syst.) } \mathrm{eV} \\
\Gamma_{1 \mathrm{~s}} & =249 \pm 111 \text { (stat.) } \pm 30 \text { (syst.) } \mathrm{eV}
\end{aligned}
$$

The shift is determined by subtracting the calculated pure electromagnetic transition energy $(6480 \pm 1 \mathrm{eV})$ from the measured X-ray transition energy. The negative sign of the shift means a repulsive strong interaction. The systematic error covers detector energy calibration, energy resolution and systematic effects due to the fitting procedure were also included. The results for the shift and width are compatible with an earlier experiment (KpX at KEK $[7,8]$ ), but our errors are significantly smaller (Fig. 4).

New theoretical studies on kaonic hydrogen recently appeared [21, 25]. One [21], using a quantum field theoretic and relativistic covariant model of the low-energy kaon-nucleon interaction near $\mathrm{K}^{-} \mathrm{p}$ threshold gives the following results for the shift $\epsilon_{1 \mathrm{~s}}=-203 \pm 15 \mathrm{eV}$ and 


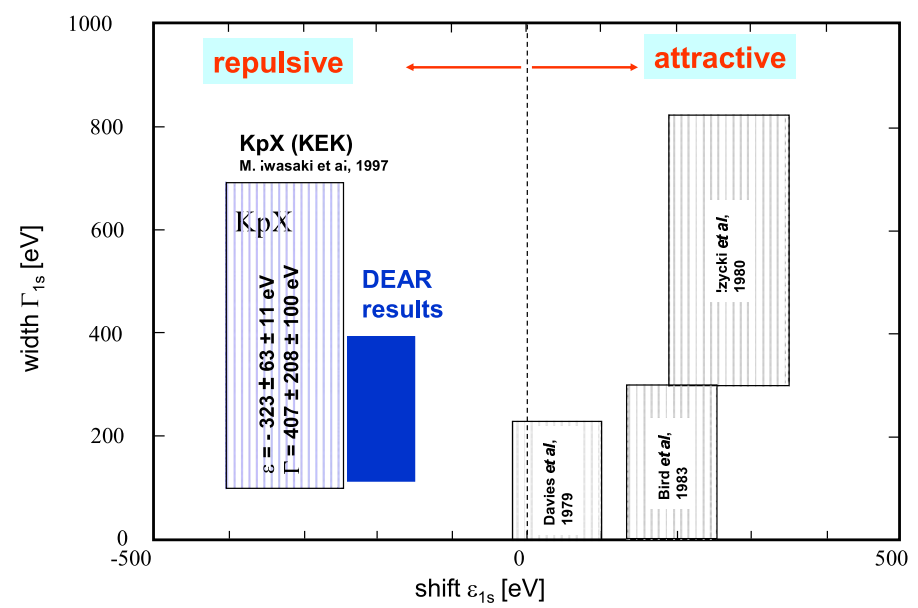

Figure 4: The DEAR result is shown in comparison with hydrogen X-ray measurements.

the width $\Gamma_{1 \mathrm{~s}}=226 \pm 28 \mathrm{eV}$, which are in good agreement with our measurement. In addition, a systematic study of corrections due to electromagnetic and QCD isospin-breaking interactions on the basis of chiral perturbation theory was published [23]. Triggered by the DEAR results on kaonic hydrogen, the chiral dynamics of kaon-nucleon interactions was revisited using chiral $\mathrm{SU}(3)$ effective field theory in combination with non-perturbative schemes based on coupled channels [25].

\section{From DEAR to SIDDHARTA}

As clearly visible in the kaonic nitrogen (Fig. 2) and kaonic hydrogen (Fig. 3a) X-ray spectra above, the background is large, although a lot of effort was put into the optimization of shielding and beam performance. The finally achieved signal-to-background ratio for kaonic nitrogen was about $1 / 10$ and 1/100 for kaonic hydrogen.

To perform precision measurements of shift and width of kaonic atoms at the percent level, the signal-to-background ratio has to be improved drastically. Therefore, we started to develop an X-ray detector, which should have good energy resolution (FWHM $\sim 150 \mathrm{eV} @$ $6 \mathrm{keV}$ ) and timing capability in the order of $\mu$ s. Using the X-ray signal from the interesting kaonic atom transition together with the $\mathrm{K}^{+} \mathrm{K}^{-}$pair, produced at DA $\Phi$ NE back-to-back in the decay of the $\Phi$-meson, a triple coincidence could be set up (see Fig. 5). With this coincidence method [26] we will suppress the continuous background, as well as fluorescence X-rays by almost three orders of magnitude compared to the DEAR case. Silicon Drift Detectors (SDDs) [27, 28] fulfill all the requirements we are looking for, except one: There were no large-area devices were available up to now (available sizes up to $10 \mathrm{~mm}^{2}$, first prototypes produced with an active area of $30 \mathrm{~mm}^{2}$ ). Therefore the SIDDHARTA 


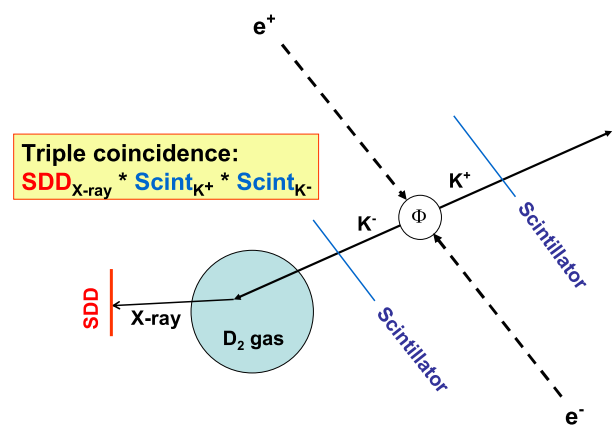

Figure 5: Schematic picture of the triple coincidence arrangement planned for the SIDDHARTA setup. The back-to-back K-pairs produced in the decay of the $\Phi$-meson will be detected by two plastic scintillators (with one scintillator directly placed in front of the target cell). In coincidence with the kaonic x-rays in the SDDs, this will strongly suppress the X-ray background compared with the DEAR case.

(Silicon Drift Detector for Hadronic Atom Research by Timing Applications) collaboration was formed within the $6^{\text {th }}$-framework program of the EU (I3-Hadron Physics), to develop large area SDD devices. The goal is to build SDD-chips with total active area of $300 \mathrm{~mm}^{2}$, consisting of 3 individual elements on one chip and finally, a detector system with a total active area of $200 \mathrm{~cm}^{2}$ is envisioned.

\section{The SIDDHARTA experimental program}

The SIDDHARTA setup (Fig. 6) follows the successful design strategy of DEAR. The cryogenic target cell will consist of a thin walled aluminum body with side windows and entrance window made out of thin Kapton foils. The SDD detector will be placed around the target with a total active detector area of about $200 \mathrm{~cm}^{2}[26]$.

\subsection{Kaonic hydrogen and deuterium}

With this setup, a percent level measurement of shift and width of kaonic hydrogen will be possible. In 30 days measuring time, with $150 \mathrm{~K}^{+} \mathrm{K}^{-}$-pairs produced per second, corresponding to an average luminosity of $10^{32} \mathrm{~cm}^{-2} \mathrm{~s}^{-1}$, more than $3 \times 10^{4}$ kaonic $\mathrm{K}_{\alpha}$ events will be collected.

Monte Carlo simulation, normalized to the measured DEAR continuous background events, shows that a first measurement of kaonic deuterium will be possible. The following assumptions, according to theory, were used in the simulation: shift $=-300 \mathrm{eV}$, width $=$ $600 \mathrm{eV}, \mathrm{X}$-ray yield $=0.2 \%$. The result is that in 30 days (with an average luminosity of $10^{32} \mathrm{~cm}^{-2} \mathrm{~s}^{-1}$ ) more than $3 \times 10^{3}$ kaonic $\mathrm{K}_{\alpha}$ events could be measured (see Fig. 7 ). 


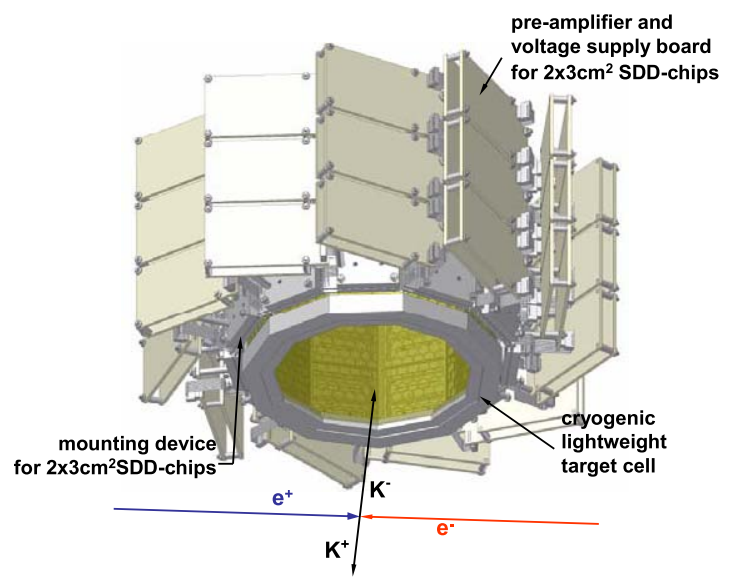

Figure 6: A possible design for SIDDHARTA is sketched. To achieve good energy resolution of the X-ray detector the SDD-chips and preamplifier electronics has to be close together. Two $3 \mathrm{~cm}^{2}$ SDD-chips are mounted in an aluminum case with the electronic board connected closely to the aluminum mount. Eleven of these units will be arranged around the target cell per row, with three rows, an active detector area of $198 \mathrm{~cm}^{2}$ will be achieved.

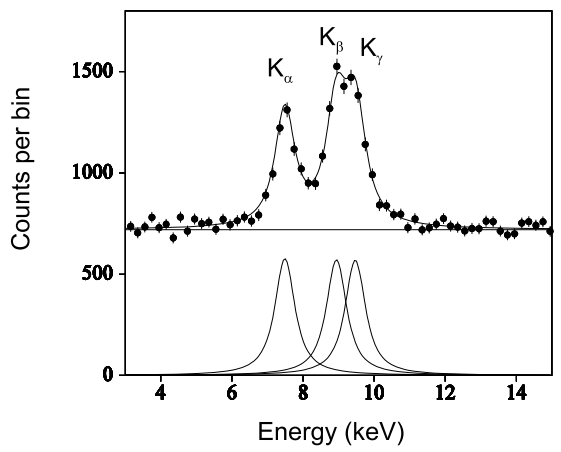

Figure 7: Monte Carlo simulation for the kaonic deuterium case with the SIDDHARTA setup.

\subsection{Kaonic helium}

It has long been known that there is a problem with the antikaon-nucleus potentials in reproducing the measured $2 \mathrm{p}$ level shift of kaonic ${ }^{4} \mathrm{He}$ atom. The measured shift and 
width (see Table 2), are both far too large to be reconciled with theoretical predictions. For example, predictions for shift and width calculated with an optical potential are [32]: $\Delta E_{\mathrm{opt} 2 \mathrm{p}}=-0.9 \mathrm{eV}$ and $\Gamma_{\mathrm{opt} 2 \mathrm{p}}=4 \mathrm{eV}$. More than 10 years ago the possibility that kaon-

Table 2: Experimental values for the strong interaction shift and width for $K^{-4} \mathrm{He}$ atoms.

\begin{tabular}{ccc}
\hline \hline$\Delta E_{2 p}(\mathrm{eV})$ & $\Gamma_{2 p}(\mathrm{eV})$ & Reference \\
\hline$-41 \pm 33$ & - & {$[33]$} \\
$-35 \pm 12$ & $30 \pm 30$ & {$[34]$} \\
$-50 \pm 12$ & $100 \pm 40$ & {$[35]$} \\
\hline \hline
\end{tabular}

nucleus bound states were formed was considered to be a way to explain the large shift [36]. A recent study by Akaishi [32] using a set of coupled-channel potentials including an antikaon-nuclear bound state indeed predicts larger shift and width of $\Delta E_{\text {c.c. } 2 \mathrm{p}}=-11 \mathrm{eV}$ and $\Gamma_{\text {c.c. } 2 \mathrm{p}}=21 \mathrm{eV}$, but these values are still small compared with the experimental results Table 2 .

Because of the large uncertainty, special in the determination of the absorption width a new measurement with better precision is urgently needed. With SIDDHARTA it will be possible not only to precisely determin the strong interaction shift and width of the L-state of kaonic ${ }^{4} \mathrm{He}$, but also to study kaonic ${ }^{3} \mathrm{He}$ for the first time.

\section{Summary}

Measurements of kaonic nitrogen and kaonic hydrogen were performed at DA $\Phi$ NE. For the first time three X-ray transition lines could be observed for kaonic nitrogen and their yield was determined. The kaonic hydrogen measurement leads to an improved accuracy in the determination of the shift and width of the ground state of kaonic hydrogen and confirms the repulsive contribution of the strong interaction (as well as the KpX experiment at KEK, Japan). Theoretical predictions based on chiral perturbation theory and quantum field theoretical approach can now be confronted with this new result.

To go further in the direction of precision spectroscopy we started to develop a new detector system - Large Area Silicon Drift Detectors -, which will allow us to perform a percent-level measurement of shift and width in kaonic hydrogen and to measure for the first time kaonic deuterium. In addition, precise measurements of kaonic ${ }^{3} \mathrm{He}$ and ${ }^{4} \mathrm{He}$ will be made with utmost precision. These measurements are of great interest for the ongoing search of deeply bound kaonic states.

\section{Acknowledgements}

The DA $\Phi$ NE group is warmly acknowledged for the excellent cooperation and teamwork. Part of the work was supported by Transnational Access to Research Infrastructure (TARI), Contract No. HPRI-CT-1999-00088. 


\section{References}

[1] S. Bianco et al., Rivista del Nuovo Cimento 22, No.11 (1999) 1

[2] Y. Nambu and G. Jona-Lasinio, Phys. Rev. 122 (1961) 345

[3] U. Vogl and W.Weise, Prog. Part. Nucl. Phys. 27 (1991) 195

[4] R. C. Carasco, Phys. Rev. C 48 (1993) 2333

[5] M. Gell-Mann and M. Levy, Nuovo Cimento 16 (1960) 706

[6] M. Gell-Mann, R.J. Oakes and B. Renner, Phys. Rev.175 (1968) 2195

[7] M. Iwasaki et al., Phys. Rev. Lett. 78 (1997) 3067

[8] T.M. Ito et al., Phys. Rev. C 58 (1998) 2366

[9] G. Beer et al., Phys. Rev. Lett. 94, 212302 (2005)

[10] J. Zmeskal et al., Hyp03-Nucl.Phys.A

[11] N. Kaiser, P.B. Siegel and W. Weise, Nucl. Phys. A 594 (1995) 325

[12] Y. Akaishi and T. Yamazaki, Phys. Rev. C 65 (2002), 44005

[13] M. Iwasaki et al. to be published and contribution in this proceedings

[14] N. Herrmann, contribution in this proceedings

[15] P. Kienle, Progress of theoretical physics supplement 149 (2003) 275

[16] see Y. Akaishi, B. Borasoy and W.Weise contributions in this proceedings

[17] T. Ishiwatari et al., Phys. Lett. B 593 ( 2004) 48

[18] PDG, Phys. Lett. B 592 (2004) 1

[19] T. Koike, contribution in this proceedings

[20] G. Beer et al., Phys. Lett. B 535 (2002) 52

[21] A. Ivanov et al., Eur. Phys. J. A21(2004) 11, nucl-th/0310081

[22] A. Ivanov et al., Eur. Phys. J. A23 (2005) 79

[23] U.-G. Meiner, U. Raha, A. Rusetsky: Eur. Phys. J. C35(2004) 349

[24] J. Gasser, Frascati Physics Series, Vol. XXXVI, Proc. of DAФNE (2004) 317

[25] B. Borasoy, R. Niler, W. Weise, Phys. Rev. Lett. 94, 213401 (2005)

[26] M. Cargnelli, in this proceedings

[27] E. Gatti, P. Rehak, Nucl. Instr. and Meth. A 225 (1984) 608

[28] J. Kemmer, G. Lutz, Nucl. Instr. and Meth. A 253 (1987) 365

[29] C.E. Wiegand and R.H. Pehl, Phys. Rev. Lett 27 (1971) 1410

[30] C.J. Batty et al., Nucl. Phys. A 326 (1979) 455

[31] S. Baird et al., Nucl. Phys. A 392 (1983) 297

[32] Y. Akaishi, private communications (2004)

[33] C.E. Wiegand and R.H. Pehl, Phys. Rev. Lett 27 (1971) 1410

[34] C.J. Batty et al., Nucl. Phys. A 326 (1979) 455

[35] S. Baird et al., Nucl. Phys. A 392 (1983) 297

[36] C.J. Batty, Nucl. Phys. A 508, (1990) 89c 